\title{
HADRON SCATTERING IN AN ADDITIVE EIKONAL QUARK MODEL
}

\author{
J. DABOUL ${ }^{1}$ and Z.J. REK ${ }^{2}$ \\ Deutsches Elektronen-Synchrotron DESY, Hamburg, Germany
}

Received 29 March 1976

Revised manuscript received 11 December 1976

\begin{abstract}
Total and differentral cross sections for $\bar{p} p, p p, \pi^{ \pm} p, K^{ \pm} p$ and $\psi p$ at high energies are analysed in an additive elkonal quark model. It is shown that the exceptionally small $\psi \mathrm{p}$ slope contradicts the Chou-Yang model at present energies but can be accounted for by a quark-quark potential with different ranges for different pairs of quarks. This leads to smaller rms radii for hadrons than those derived from e.m. formfactors, implying that the constituent quarks have formfactors. Predictions for hadron-neutron and strange and charmed hadron-nucleon scattering are derived.
\end{abstract}

The purpose of this letter is to extend the additive quark model, which has been quite successful in relating total hadronic cross sections [1-3], to describe the differential cross sections as well. Our model, besides being phenomenologically very useful, gives information on the structure of hadrons and on quark-quark interactions. While the systematics of total cross sections $\sigma_{\overline{\mathrm{pp}}}>\sigma_{\mathrm{pp}}>\sigma_{\pi^{-} \mathrm{p}}>\sigma_{\pi^{+} \mathrm{p}}>\sigma_{\mathrm{K}^{-} \mathrm{p}}>\sigma_{\mathrm{K}_{\mathrm{p}}^{+}}>\sigma_{\phi \mathrm{p}}>\sigma_{\psi \mathrm{p}}$ could be simply attributed to different strengths of quark-quark scattering amplitudes, a more complicated mechanism is necessary to explain the identical inequalities among the forward slopes of differential cross sections, $B_{\overline{\mathrm{p} p}}>B_{\mathrm{pp}}>\ldots$ etc.

Unlike the usual additive quark model we assume additivity at the level of eikonals ${ }^{{ }^{1}}$ rather than amplitudes [4], i.e.

$$
\chi^{\mathrm{AB}}(s, b)=\sum_{i, j=1}^{2 N} n_{i}^{\mathrm{A}} n_{j}^{\mathrm{B}} \chi_{i j}^{\mathrm{AB}}(s, b),
$$

where $n_{i}^{\mathrm{A}}$ is the number of quarks (antiquarks) of type $i$ in particle $\mathrm{A}$ and $N$ is the number of flavours. This "additive eikonal quark model" could, for example, be justified using a potential model in e1konal approximation in which case $\chi_{i j}^{\mathrm{AB}}$ is given by

$$
\chi_{i j}^{\mathrm{AB}}(s, b)=\int_{-\infty}^{\infty} \mathrm{d} z \int \mathrm{d}^{3} r_{1} \mathrm{~d}^{3} r_{2} \rho_{\mathrm{A}}\left(r_{1}\right) \rho_{\mathrm{B}}\left(r_{1}\right) \rho_{\mathrm{B}}\left(r_{2}\right) I_{i j}^{\mathrm{AB}}\left(s, r-r_{1}+r_{2}\right) .
$$

Here $\rho_{\mathrm{h}} \neq^{2}$ is the density distribution of quarks in hadron $\mathrm{h}$ and $I_{i j}^{\mathrm{AB}}$ is the effective quark-quark potential, which may also depend on the interacting hadrons, as will be discussed later. If we classify the quark eikonals similarly to Lipkin [2]

1 Permanent address: Physics Department, Ben Gurion University, Beer Sheva, Israel.

2 On leave of absence from Institute of Nuclear Research, Warsaw, Poland.

$\neq^{1}$ The eikonal $\mathrm{x}(s, b)$ is defined by the usual relation

$$
\mathrm{d} \sigma \mathrm{AB} / \mathrm{d} t=\pi\left|\int_{0}^{\infty} b \mathrm{~d} b J_{0}(b v-t)\left(1-\exp \left\{-\chi^{\mathrm{AB}}(s, b)\right\}\right)\right|^{2} .
$$

$\neq^{2}$ From now on we shall use the following subscripts: $h$ - hadron, $m-$ meson, $b$ - baryon, $a-$ annihilation, $d-$ non strange, c - charm. 


$$
\begin{array}{ll}
\chi_{\mathrm{d} \overline{\mathrm{d}}}=\chi_{\mathrm{u} \overline{\mathrm{u}}} \equiv \chi_{\mathrm{a}}, & \chi_{\mathrm{uu}}=\chi_{\mathrm{dd}}=\chi_{\mathrm{ud}}=\chi_{\mathrm{u} \overline{\mathrm{d}}} \equiv \chi_{\mathrm{d}}, \\
\chi_{\mathrm{sd}}=\chi_{\overline{\mathrm{s} d}}=\chi_{\mathrm{su}}=\chi_{\overline{\mathrm{s} u}} \equiv \chi_{\mathrm{s}}, & \chi_{\mathrm{cd}}=\chi_{\overline{\mathbf{c} d}}=\chi_{\mathrm{cu}}=\chi_{\overline{\mathrm{c} u}} \equiv \chi_{\mathrm{c}},
\end{array}
$$

and assume that $\chi_{\mathrm{a}, \mathrm{d}, \mathrm{s}, \mathrm{c}}$ are independent of $\mathrm{AB}$, we get immediately Lipkin's sum rules but at the level of eikonals instead of total cross sections. Unfortunately, the eikonal is rather difficult to calculate directly from experiment [5]; in particular, $\mathrm{d} \sigma / \mathrm{d} t$ must be known for large $|t|$ and with high accuracy. Therefore, instead of checking the eikonal relations directly, we prefer to parametrize the eikonals and compare the resulting differential and total cross sections with experiment.

Approximating the scattering amplitude, for simplicity, by a purely imaginary exponentral, $f=\mathrm{i} \sigma^{\text {tot }} \exp (B t / 2) /$ $(4 \sqrt{\pi})$, gives

$$
\frac{\partial \tilde{\chi}(s, t)}{\partial t} /\left.\widetilde{\chi}(s, t)\right|_{t=0}=\frac{1}{2} B g(Y), \quad Y_{\mathrm{AB}} \equiv \sigma_{\mathrm{AB}}^{\text {tot }} / 4 B_{\mathrm{AB}}
$$

with $g(Y) \equiv\left(\sum_{n=1}^{\infty} Y^{n} / n^{2}\right) /\left(\Sigma_{n=1}^{\infty} Y^{n} / n^{3}\right)$; here $\tilde{\chi}(s, t)$ is the Fourier-Bessel transform of the eikonal. On the other hand, from eq. (2) we get

$$
\tilde{\chi}_{i j}^{\mathrm{AB}}(s, t)=\widetilde{\rho}_{\mathrm{A}}(t) \tilde{\rho}_{\mathrm{B}}(t) I_{l j}^{\mathrm{AB}}(s, t),
$$

so that (in case $\chi_{\mathrm{AB}} \propto \chi_{l j}^{\mathrm{AB}}$ with one type of $\chi_{l j}$ )

$$
B_{\mathrm{AB}}=\frac{1}{3} g\left(Y_{\mathrm{AB}}\right)\left(\left\langle r_{\mathrm{A}}^{2}\right\rangle+\left\langle\nabla_{\mathrm{B}}^{2}\right\rangle+\left\langle R_{i j}^{2}\right\rangle\right),
$$

where " $\sim$ " denotes the 3-dim. Fourier transform $\left(t=-\Delta^{2}\right),\left\langle r_{A}^{2}\right\rangle$ is the mean square radius of particle $A$ and $\left\langle R_{i j}^{2}\right\rangle$ $=\int r^{2} I_{i j}(r) \mathrm{d}^{3} r / \int I_{i j}(r) \mathrm{d}^{3} r$ is a measure of quark-quark interaction range. In the high energy region, where $Y$ $\equiv \sigma^{\text {tot }} / 4 \pi B<1$ for all hadronic reactions, $g(Y)$ is a monotonic function, satisfying $1=g(0) \leqslant g(Y) \leqslant g(1)=1.368$.

Applying (6) simultaneously to $\psi \mathrm{p}$ and pp and using $g\left(Y_{\psi \mathrm{p}}\right) \simeq 1.0, g\left(Y_{\mathrm{pp}}\right) \simeq 1.18$ and $B_{\mathrm{pp}} \simeq 9.6 \mathrm{GeV}^{-2}$ (for $\left.p_{\text {lab }}=50 \mathrm{GeV} / c\right)$, gives

$$
\left\langle v_{\psi}^{2}\right\rangle \simeq 3\left[B_{\psi \mathrm{p}} / g\left(F_{\psi \mathrm{p}}\right)-\frac{1}{2} B_{\mathrm{pp}} / g\left(F_{\mathrm{pp}}\right)\right]+\frac{1}{2}\left\langle R_{\mathrm{d}}^{2}\right\rangle-\left\langle R_{\mathrm{c}}^{2}\right\rangle \simeq 3\left(B_{\psi \mathrm{p}}-4.1 \mathrm{GeV}^{-2}\right)+\frac{1}{2}\left\langle R_{\mathrm{d}}^{2}\right\rangle-\left\langle R_{\mathrm{c}}^{2}\right\rangle,
$$

where we used $\left\langle R_{\mathrm{dd}}^{2}\right\rangle=\left\langle R_{\mathrm{du}}^{2}\right\rangle \equiv\left\langle R_{\mathrm{d}}^{2}\right\rangle$ and $\left\langle R_{\mathrm{cd}}^{2}\right\rangle=\left\langle R_{\mathrm{cd}}^{2}\right\rangle \equiv\left\langle R_{\mathrm{c}}^{2}\right\rangle$ (see (3)). Hence, the Chou-Yang model [6] with contact quark-quark interaction, $I_{\mathrm{qq}}(r) \propto \delta(r)$ or, equivalently, $\left\langle R_{\mathrm{d}}^{2}\right\rangle=\left\langle R_{\mathrm{c}}^{2}\right\rangle=0$, would lead to negative $\left\langle r_{\psi}^{2}\right\rangle$ as long as $B_{\psi \mathrm{p}}<4.1 \mathrm{GeV}^{-2}$. Even the largest slope of $B_{\psi \mathrm{p}}=2.9 \pm 0.3$ [8], deduced from photoproduction experiments up to $E_{\gamma} \simeq 100 \mathrm{GeV}$, leads to a contradiction.

One possibe way out is to assume that different pairs of quarks have different interaction ranges, in particular $\left.\left\langle R_{\mathrm{d}}^{2}\right\rangle\right\rangle 2\left\langle R_{\mathrm{c}}^{2}\right\rangle$. However, since $\left\langle\gamma_{\mathrm{p}}^{2}\right\rangle \simeq \frac{3}{2} B_{\mathrm{pp}} / g\left(Y_{\mathrm{pp}}\right)-\frac{1}{2}\left\langle R_{\mathrm{d}}^{2}\right\rangle=(0.7)^{2} \mathrm{fm}^{2}-\frac{1}{2}\left\langle R_{\mathrm{d}}^{2}\right\rangle$, a large $\left\langle R_{\mathrm{d}}^{2}\right\rangle$ would lead to a smaller rms radius for proton than the one following from its e.m. formfactor, $6 \mathrm{~d} F(t) /\left.\mathrm{d} t\right|_{t=0} \simeq(0.8)^{2} \mathrm{fm}^{2}$. To account for the difference one has to assume that constituent quarks themselves have their own formfactors. Then the observed e.m. formfactor of hadron $F_{\mathrm{h}}$ would be given roughly by the product $Q_{\mathrm{h}} F_{\mathrm{h}}(t) \simeq \widetilde{\rho}(t) \Sigma Q_{\mathrm{q}} F_{\mathrm{q}}(t)$, where $Q_{\mathrm{q}}$ are the charges of the constituent quarks and $F_{\mathrm{q}}(t)$ are their e.m. formfactors. This point will be discussed in more detail elsewhere [7].

To test this Idea we use the following simple parametrization of the density distribution and quark-quark effective potential

$$
\begin{aligned}
& \rho_{\mathrm{A}}(r)=\pi^{-3 / 2} r_{\mathrm{A}}^{-3} \exp \left(-r^{2} / r_{\mathrm{A}}^{2}\right), \\
& I_{i j}^{\mathrm{AB}}(s, r)=\gamma^{\mathrm{AB}}(s) \lambda_{i j}(s) \exp \left(-r^{2} / R_{i j}^{2}(s)\right)+\gamma_{\text {core }}^{\mathrm{AB}}(s) \lambda_{i j}^{\text {core }}(s) \exp \left(-r^{2} / R_{\text {core }, i j}^{2}(s)\right) .
\end{aligned}
$$




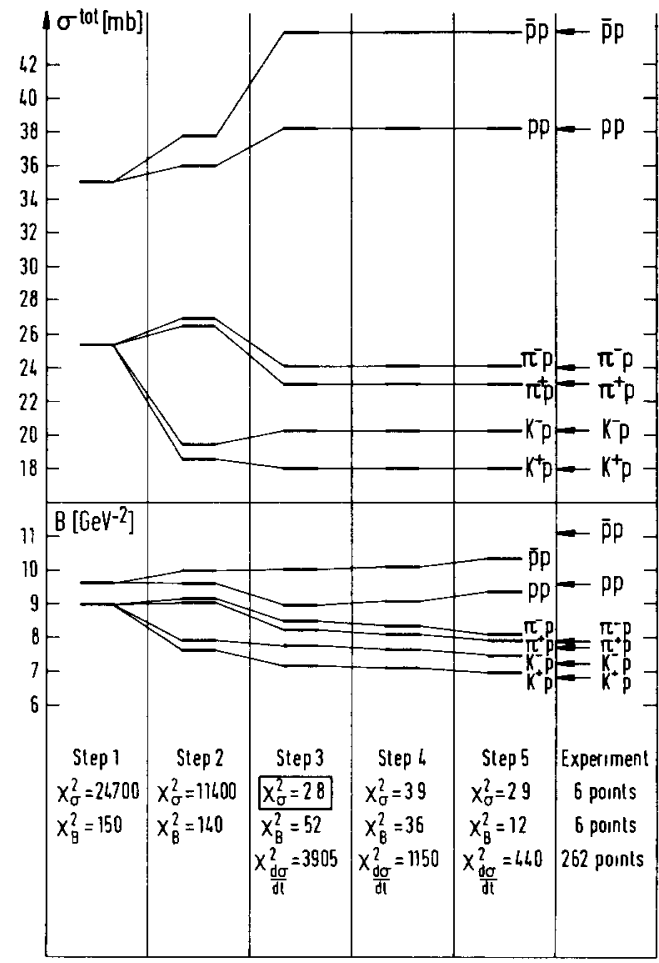

Fig. 1. Schematic improvement of the successive steps of our

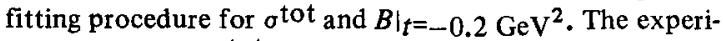
mental values of $\sigma^{\text {tot }}$ and $B$ are given in table 1 .

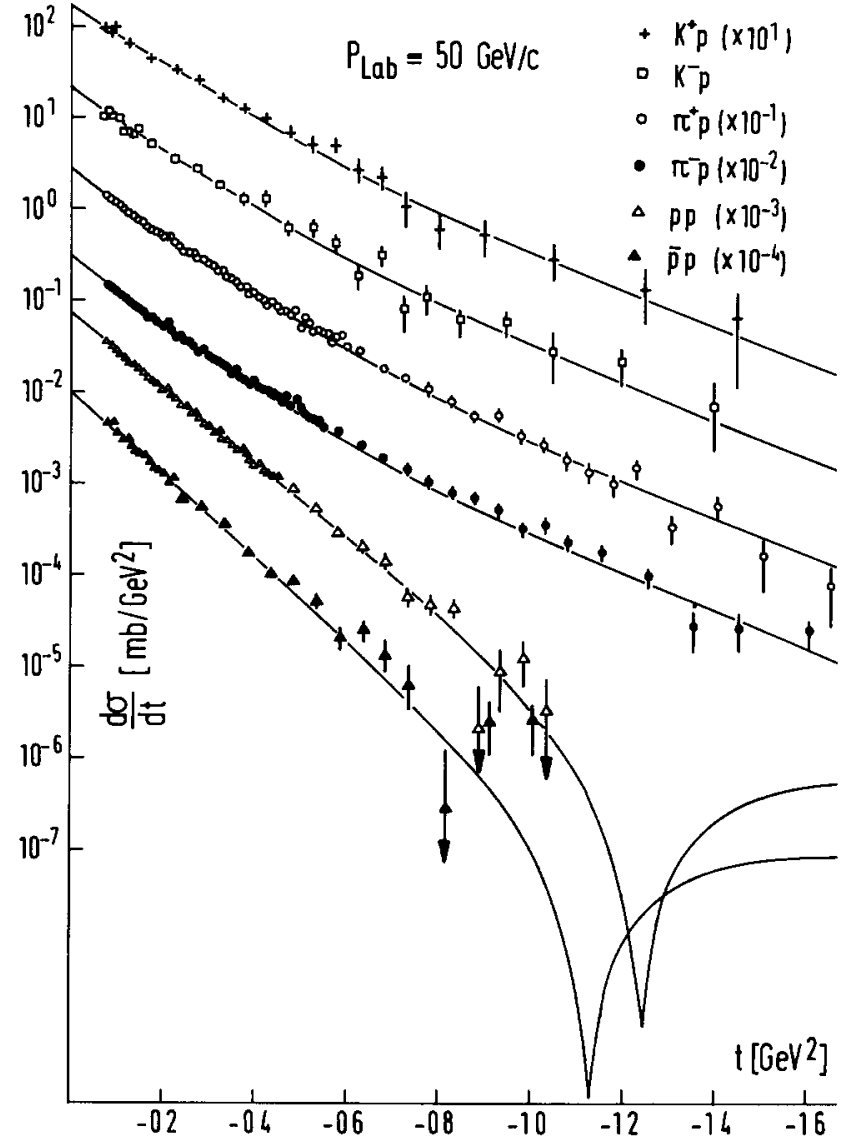

Fig. 2. The differential cross sections for elastic scattering of $\mathrm{K}^{ \pm}, \pi^{ \pm}, \mathrm{p}$ and $\overline{\mathrm{p}}$ on protons at $p_{\mathrm{lab}}=50 \mathrm{GeV} / c$. The curves are the result of our best fit (step 5). The experimental data are taken from ref. [11].

The corresponding eikonals are

$$
\chi_{i j}^{\mathrm{AB}}(s, b)=g_{i j}^{\mathrm{AB}}(s) \exp \left(-b^{2} / \bar{R}_{i j}^{2}\right)+g_{\text {core }, i j}^{\mathrm{AB}}(s) \exp \left(-b^{2} / \bar{R}_{\text {core }, i j}^{2}\right),
$$

where

$$
\begin{aligned}
& g_{i j}^{\mathrm{AB}}=\gamma^{\mathrm{AB}} \lambda_{l j} \frac{\sqrt{\pi} R_{t j}^{3}}{r_{\mathrm{A}}^{2}+r_{\mathrm{B}}^{2}+R_{i j}^{2}}, \\
& \bar{R}_{i j}^{2}=r_{\mathrm{A}}^{2}+r_{\mathrm{B}}^{2}+R_{i j}^{2},
\end{aligned}
$$

and similarly for the core.

To explain the meaning and the role of different parameters in the above ansatz, we shall start by assuming drastic simplifying relations among them and then relax some of these gradually, showing the improvement of the 
Table 1

The total cross sections $\sigma^{\text {tot }}$ and the slopes $B$ (calculated at $t=-0.2 \mathrm{GeV}^{2}$ ) at $p_{\mathrm{lab}}=50 \mathrm{GeV} / c$. The upper part of the table includes the input data, the lower one, predictions. The nomenclature of the charmed particles (except $\psi$ ) corresponds to the one in ref. [19].

\begin{tabular}{|c|c|c|c|c|c|c|}
\hline Reaction & $\sigma_{\mathrm{th}}^{\mathrm{tot}}(\mathrm{mb})$ & $\sigma_{\exp }^{t o t}(m b)$ & Ref. & $B_{\mathrm{th}}\left(\mathrm{GeV}^{-2}\right)$ & $B_{\exp }\left(\mathrm{GeV}^{-2}\right)$ & Ref. \\
\hline$\overline{\mathrm{p}} \mathrm{p}$ & 43.99 & $43.93 \pm 0.10$ & 12 & 10.35 & $11.13 \pm 0.30$ & 11 \\
\hline $\mathrm{p} p$ & 38.23 & $38.20 \pm 0.05$ & 12 & 9.38 & $9.61 \pm 0.15$ & 11 \\
\hline$\pi \bar{p}$ & 24.14 & $24.06 \pm 0.06$ & 12 & 8.13 & $7.92 \pm 0.17$ & 11 \\
\hline$\pi^{+} p$ & 23.07 & $23.11 \pm 0.06$ & 12 & 7.92 & $7.74 \pm 0.17$ & 11 \\
\hline $\mathrm{K}^{-} \mathrm{p}$ & 20.30 & $20.30 \pm 0.10$ & 12 & 7.49 & $7.26 \pm 0.37$ & 11 \\
\hline $\mathrm{K}^{+} \mathrm{p}$ & 18.06 & $18.06 \pm 0.08$ & 12 & 6.98 & $6.86 \pm 0.45$ & 11 \\
\hline$\psi \mathrm{p}$ & 1.69 & $1.0-2.75$ & $8-10$ & 2.9 & $1.8-2.9$ & $8-10$ \\
\hline$\overline{\mathbf{p}} \mathrm{n}$ & 42.86 & $43.88 \pm 0.21$ & 12 & \multirow[t]{2}{*}{10.16} & & \\
\hline \multirow{2}{*}{ p n } & \multirow{2}{*}{38.23} & $\begin{array}{l}42.5 \pm 1.0 \\
38.96 \pm 0.09\end{array}$ & $\begin{array}{l}13 \\
12\end{array}$ & & & \\
\hline & & $38.26 \pm 0.25$ & 13 & 9.38 & & \\
\hline $\mathrm{K}^{-} \mathrm{n}$ & 19.18 & $19.86 \pm 0.16$ & 12 & \multirow{2}{*}{7.25} & & \\
\hline \multirow{2}{*}{$\mathrm{K}^{+} \mathrm{n}$} & \multirow{2}{*}{18.06} & $\begin{array}{l}19.41 \pm 0.15 \\
18.66 \pm 0.14\end{array}$ & 13 & & & \\
\hline & & $\begin{array}{l}18.66 \pm 0.14 \\
18.31 \pm 0.17\end{array}$ & $\begin{array}{l}12 \\
13\end{array}$ & 6.98 & & \\
\hline$\phi \mathrm{p}$ & 13.92 & $7.7-8.7^{\mathrm{a}}$ & 14 & 6.06 & $5.04-5.97^{\mathrm{a}}$ & 14 \\
\hline$\Lambda p$ & 34.04 & $34.6 \pm 0.4^{b}$ & 15 & 8.75 & & \\
\hline$\Lambda \mathrm{n}$ & 34.04 & $34.0 \pm 0.4^{b}$ & 15 & 8.75 & & \\
\hline $\bar{\Lambda} \mathrm{p}$ & 37.70 & $49.3 \pm 3.7^{c}$ & 16 & 9.38 & & \\
\hline $\bar{\Lambda} \mathrm{n}$ & 37.70 & $46 \pm 20 \mathrm{~b}$ & 15 & 9.38 & & \\
\hline$\Sigma^{-} p$ & 34.04 & $34.0 \pm 1.1 \mathrm{~d}$ & 17 & 8.75 & & \\
\hline$\Sigma^{-} n$ & 34.04 & $30.0 \pm 1.2^{\mathrm{d}}$ & 17 & 8.75 & & \\
\hline $\mathrm{D}^{+} \mathrm{p}$ & 13.87 & & & 7.18 & & \\
\hline$D^{0} p$ & 15.07 & & & 7.50 & & \\
\hline$D^{-} p$ & 12.65 & & & 6.83 & & \\
\hline $\mathrm{F}^{+} \mathrm{p}$ & 8.16 & & & 5.46 & & \\
\hline$C q p$ & 28.59 & & & 8.49 & & \\
\hline $\mathrm{S}_{\mathrm{p}}^{+}$ & 23.76 & & & 7.68 & & \\
\hline $\mathrm{T}^{0} \mathrm{p}$ & 18.03 & & & 6.68 & & \\
\hline $\mathrm{x}_{\mathrm{d}}^{+} \mathrm{p}$ & 17.13 & & & 7.34 & & \\
\hline$X_{s}^{+} p$ & 11.48 & & & 5.91 & & \\
\hline
\end{tabular}

\footnotetext{
a Measured over $p_{\mathrm{lab}}=4.6-6.7 \mathrm{GeV} / c$.

$\mathrm{b}$ Measured over $p_{\mathrm{lab}}=6-21 \mathrm{GeV} / c$.

c Measured over $p_{\mathrm{lab}}=4-14 \mathrm{GeV} / c$.

$\mathrm{d}$ Measured for $p_{\text {lab }}=18.7 \mathrm{GeV} / c$.
}

fit as the number of parameters grows. We base our fit on 6 reactions, $\bar{p} p, p p, \pi^{ \pm} p$ and $K^{ \pm} p$, and for every one of them we use 6 data points: $\sigma^{\text {tot }}, B$ (with additional weight 9 to give $B$ equal footing with $\sigma^{\text {tot }}$ in the fit) and 4 points of $\mathrm{d} \sigma / \mathrm{d} t$.

In fig. 1 we 1 llustrate the improvement of our fit by plotting $\sigma^{\text {tot }}$ and $B$ together with $\chi^{2}$ for all fitting steps and in fig. 2 the differential cross sections resulting from the final fit. Now, we shall shortly review successive approximations $\ddagger^{\ddagger^{3}}$.

$f^{3}$ Note that the first two steps correspond to naive [1] and sophisticated [2] quark model, while the other 3 steps are the natural extension of the former, indicated by experiment. 
Step 1 (2 parameters):

$$
\begin{array}{ll}
\lambda_{i j}=\lambda, R_{i j}=R, \lambda_{i j}^{\text {core }}=0 & \text { for all } i, j ; \\
\gamma_{\mathrm{AB}}=1, r_{\mathrm{A}}=r_{\mathrm{B}}=r_{\mathrm{h}}=0.28 \mathrm{fm} & \text { for all } \mathrm{A}, \mathrm{B} .
\end{array}
$$

The above common value $r_{\mathrm{h}}$ for all hadrons, corresponding to a rms radius $\sqrt{\left\langle r_{\mathrm{h}}^{2}\right\rangle}=\sqrt{1.5 r_{\mathrm{h}}^{2}}=0.34 \mathrm{fm}$, is chosen as large as possible within the limits imposed by $B_{\psi \mathrm{p}} \neq^{4}$. These assumptions leave us with 2 parameters only, $R$ and $\lambda$, but already at this stage the quark counting rule at the eikonal level, $\chi_{\mathrm{bb}}=\frac{9}{6} \chi_{\mathrm{mb}}$, gives a splitting of both $\sigma^{\text {tot }}$ and $B[4]$ into common values for mesons and baryons.

Step 2 (4 parameters). Allow for different ranges of quark interactions according to classification (3): $R_{\mathrm{a}}>R_{\mathrm{d}}$ $>R_{\mathrm{s}}$. In particular, the inequality $R_{\mathrm{a}}>R_{\mathrm{d}}$ leads to $\overline{\mathrm{p}} \mathrm{p}>\mathrm{pp}, \pi^{-} \mathrm{p}>\pi^{+} \mathrm{p}, \mathrm{K}^{-} \mathrm{p}>\mathrm{K}^{+} \mathrm{p}$ for both $\sigma^{\text {tot }}$ and $B$. Note that although $\lambda$ is still common for all quarks, the corresponding $g$ 's will split, $g_{\mathrm{a}}>g_{\mathrm{d}}>g_{\mathrm{s}}$.

Step 3 (5 parameters). The observation that $\sigma_{\mathrm{b}}^{\text {tot }}$ is always smaller whereas $\sigma_{\mathrm{m}}^{\text {tot }}$ is always larger than experiment (see fig. 1) lead us to introduce a phenomenological parameter $\gamma_{b}>\gamma_{m} \equiv 1$. This parameter is responsible for the difference between effective quark-quark couplings in meson-baryon and baryon-baryon scattering and could be attributed to the dependence of quark interactions on velocity and different velocity distributions of quarks within mesons and baryons. If this is the case, then $\gamma$ should approach unity as $s \rightarrow \infty$. The additional parameter $\gamma_{\mathrm{b}}$ improves the fit enormously (compare $\chi^{2}$ in fig. 1 ).

Step 4 ( 7 parameters). Although the above 5 parameter fit leads to very good results for $\sigma^{\text {tot }}$ and $B$, the theoretical differential cross sections show a prominent dip at too low values of $t\left(\simeq-1 \mathrm{GeV}^{2}\right)$ for all 6 reactions. By introducing a core term, with $R_{\text {core, } i j} \ll R_{i j}$, we can push the dip to larger values of $t$. For simplicity we add only 2 new core parameters: common coupling $\lambda$ core and a common ratio $\alpha=R_{\text {core, } \mathrm{a}}^{2} / R_{\mathrm{a}}^{2}=R_{\text {core, } \mathrm{d}}^{2} / R_{\mathrm{d}}^{2}=R_{\text {core, } \mathrm{s}}^{2} / R_{\mathrm{s}}^{2}$ and assume $\gamma_{\mathrm{b}}^{\text {core }}=\gamma_{\mathrm{b}}, \gamma_{\mathrm{m}}^{\text {core }}=1$.

Step 5 ( 7 parameters, $r_{\mathrm{m}} \neq r_{\mathrm{b}}$ ). The calculated baryon (meson) slopes are always smaller (larger) than the experımental values. This implies in our model that baryons have larger radii than mesons. Unfortunately, due to the limited range in $t$ of present $\mathrm{pp}$ and $\overline{\mathrm{p}} \mathrm{p}$ data, from which no conclusion about the existence or position of the dip may be drawn, our fit is not very sensitive to the change of $r_{m}$ and $r_{b}$. Therefore, we repeated step 4 with somewhat arbitrarily fixed values $r_{\mathrm{m}}=0.24 \mathrm{fm}$ and $r_{\mathrm{b}}=0.32 \mathrm{fm}$. This gives an excellent agreement with all present data (see figs. 1,2 and table 1). As we checked, the dip in pp can be pushed to larger momentum transfers by changing parameters $r_{\mathrm{m}}, r_{\mathrm{b}}$ and $\alpha$ without substantial worsening of other reactions. The values of parameters obtained are: $R_{\mathrm{a}}=0.81 \mathrm{fm}, R_{\mathrm{d}}=0.73 \mathrm{fm}, R_{\mathrm{s}}=0.62 \mathrm{fm}, \lambda=0.0156 \mathrm{GeV}, \gamma_{\mathrm{b}}=1.318, \alpha=0.119, \lambda^{\text {core }}=0.121 \mathrm{GeV}$. Note that $\lambda$ couplings are real which corresponds to purely absorptive quark-quark potential.

For $\psi \mathrm{p}$ the experimental data have large uncertainties [8-10]: $1.25 \pm 0.2<B_{\psi \mathrm{p}}<2.9 \pm 0.3 \mathrm{GeV}^{-2}$ and $1<\sigma_{\psi \mathrm{p}}^{\text {tot }}<3 \mathrm{mb}$. To estimate the one additional charmed quark parameter, $R_{\mathrm{c}}$, we used $B_{\psi \mathrm{p}}=2.9 \mathrm{GeV}^{-2}$. Then $\sigma_{\psi \mathrm{p}}^{\text {tot }}$ is predicted to be $1.69 \mathrm{mb}$ and $R_{\mathrm{c}}=0.30 \mathrm{fm}$.

Finally, we list in table 1 predictions for the total cross sections and slopes for several reactions and compare them with experiment when possible. Note the remarkable agreement with the experimental neutron data: less than $2 \%$ deviation from Fermilab data [12] and even better agreement with Serpukhov data [13]. Unfortunately, the hyperon-nucleon scattering is measured only up to $p_{\text {lab }}=21 \mathrm{GeV} / c$ so that direct comparison is not possible. However, the rough agreement is visible if one remembers that $\bar{\Lambda}_{p}$ and $\bar{\Lambda}_{n}$ decrease faster with energy than $\Lambda p$ and $\Lambda \mathrm{n}$, due to the strongly varying annihilation channel $\overline{\mathrm{d}} \mathrm{d}$ and $\overline{\mathrm{u}} \mathrm{u}$. The predictions for charmed particles are intended only as rough estımates.

We conclude that the additive eikonal quark model describes successfully the different hadron scattering data. Our good fit shows implicitly that the quark model relations (3) are well satisfied within mesons and baryons respectively and indicates the necessity of breaking of simple additivity by introducing some additional difference be-

$\neq^{4}$ The distribution $\rho_{\mathrm{h}}$ for different quarks should be unequal if some quarks are much heaver than the others (e.g. charmed quark). Never theless, we use a universal $\rho_{\mathbf{h}}$ for simplicity. 
tween mesons and baryons $\left(\gamma_{\mathrm{b}}\right.$ parameter). The interpretation given to the various parameters has important implications for the quark dynamics and the structure of hadrons. To gain a deeper understanding of this model we used it also to study the energy dependence of hadronic interactions. The results will be discussed elsewhere [18].

It is a pleasure to thank Dr. F. Gutbrod, Prof. H. Joos, Dr. M. Krammer, Dr. M. Kuroda and Dr. T. Walsh for very fruitful discussions.

\section{References}

[1] E.M. Levein and L.L. Frankfurt, JETP Lett. 2 (1965) 65;

H.J. Lipkin and F. Scheck, Phys. Rev. Lett. 16 (1966) 336.

[2] H.J. Lipkin, Phys. Rev. Lett. 16 (1966) 1015.

[3] H.J. Lipkin, Phys. Rev. D11 (1975) 1827.

[4] The additivity of eikonals has been discussed for equal quark-quark eikonals (step 1 in our paper) by J. Dras de Deus, Acta Phys. Polonica B6 (1975) 613;

J. Fröyland, Phys. Lett. 58B (1975) 317.

[5] H. Mettinnen, IX Rencontre de Moriond (1974).

[6] T.T. Chou and C.N. Yang, Phys. Rev. Lett. 20 (1968) 1213, Phys. Rev. 170 (1968) 1591;

L. Durand III and R. Lipes, Phys. Rev. Lett. 20 (1968) 637;

T. Inami, Argonne preprint ANL-HEP-PR-75-42;

V. Franco, Phys. Rev. D1 1 (1975) 1837;

Y. Suzuki and M. Yonezawa, Lett. Nuovo Cim. 14 (1975) 267.

[7] J. Daboul, in preparation.

[8] R. Prepost, SLAC-PUB-1689 (1975).

[9] T. Nash et al., Fermilab-Pub-76/25-EXP (1976).

[10] R.L. Anderson, SLAC-PUB-1741 (1976).

[11] C.W. Akerlof et al., Michıgan preprint UM HE 76-6 (1976).

[12] A.S. Carroll et al., Phys. Lett. 61 B (1976) 303.

[13] S.P. Denisov et al., Nucl. Phys. B65 (1973) 1.

[14] H.-J. Behrend et al., Phys. Lett. 56B (1975) 408.

[15] S. Gjesdal et al., Phys. Lett. 40B (1972) 152.

[16] F. Eisele et al., Phys. Lett. 60B (1976) 297.

[17] J. Badier et al., Phys. Lett. 41B (1972) 387.

[18] J. Daboul and Z.J. Rek, in preparation.

[19] M.K. Gallard, B. Lee and J. Rosner, Rev. Mod. Phys. 47 (1975) 277. 